\title{
Success Story of a Low Cost Intra-Dermal Rabies Vaccination (IDRV) Clinic-Lessons Learnt over Five Years of 12,000 Patient Vaccinations "Without Failure" at DDU Hospital Shimla, Himachal Pradesh, India
}

\author{
—“Saving a Drop of Rabies Vaccine and Immunoglobulins" 12 \\ Innovations to Make Himachal Pradesh Rabies Free State by 2020
}

\author{
Omesh Kumar Bharti1 ${ }^{*}$, Shampur Narayan Madhusudana², Ashok Kale 3 , Pyare Lal Gaunta4, \\ Laxman Singh Chaudhry ${ }^{5}$, Jitender Kumar ${ }^{4}$, Nirmal Gupta ${ }^{6}$, Dropda Shyam ${ }^{7}$ \\ ${ }^{1}$ Faculty Epidemiologist, State Institute of Health \& Family Welfare, Parimahal, Shimla, India \\ ${ }^{2}$ Professor of Neurovirology, Head, WHO Collaborating Centre for Reference and Research on Rabies, \\ Department of Neurovirology, NIMHANS, Bangalore, India \\ ${ }^{3}$ Professor, Department of Community Medicine, Dr. Ulhas Patil Medical College \& Hospital, Jalgaon, India \\ ${ }^{4}$ Rtd. Sr. Medical Superintendent, DDU Hospital, Shimla, India \\ ${ }^{5}$ Associate Professor, Department of Pediatrics, Indira Gandhi Medical College, Shimla, India \\ ${ }^{6}$ Staff Nurse, Anti-Rabies Clinic and Research Centre (ARC\&RC), DDU Hospital, Shimla, India \\ ${ }^{7}$ Multi Purpose Health Worker (MPW) previously at Anti-Rabies Clinic and Research Centre, DDU Hospital, \\ Shimla, India \\ Email: "bhartiomesh@yahoo.com
}

Received 9 May 2015; accepted 17 July 2015; published 20 July 2015

Copyright (C) 2015 by authors and Scientific Research Publishing Inc.

This work is licensed under the Creative Commons Attribution International License (CC BY).

http://creativecommons.org/licenses/by/4.0/

(c) (i) Open Access

\section{Abstract}

Background: Rabies is a dreaded disease and an estimated 55,000 people die of rabies every year. Himachal Pradesh is in the North bordering China and is predominantly rural and hilly. Villages are near forests, where wild reservoirs of rabies exist. Since health facilities are not accessible easily, we need to innovate on existing schedules of rabies vaccination keeping in view the compliance of the patients and affordability so as to give them the best possible option of treatment. In the year 2006 and 2007, we, at DDU Hospital Shimla, experienced a severe shortage of rabies vaccine and patients were running from pillar to post to fetch rabies vaccine. At the same time, we

*Corresponding author.

How to cite this paper: Bharti, O.K., Madhusudana, S.N., Kale, A., Gaunta, P.L., Chaudhry, L.S., Kumar, J., Gupta, N. and Shyam, D. (2015) Success Story of a Low Cost Intra-Dermal Rabies Vaccination (IDRV) Clinic-Lessons Learnt over Five Years of 12,000 Patient Vaccinations "Without Failure" at DDU Hospital Shimla, Himachal Pradesh, India. World Journal of Vaccines, 5, 129-139. http://dx.doi.org/10.4236/wjv.2015.53014 
learnt that some of the patients died because either they were not able to purchase the vaccine, mostly because of its high cost, $\$ 35$, or they ignored the animal bites and did not seek the treatment. Since last year, we have been experiencing non-availability of rabies immunoglobulins (RIGs) in the market and have to innovate new schedules and techniques to save lives of the patients. Methods: During shortage of rabies vaccine in 2008, we contemplated to start a low cost intra-dermal (ID) clinic so as to make rabies vaccine affordable as intramuscular (IM) vaccination cost five times more than ID vaccination. But, there were three main hurdles. One hurdle was the non-availability of rabies vaccine vials having written on them "For IM/ID use" and another hurdle was only fewer animal bite patients attending the DDU Hospital, sometimes only one or two per day, which was insufficient to open a vaccine vial and distribute among them. The third problem being faced was reluctance of the hospital doctors to prescribe ID vaccine as this was not the practice at higher teaching institutions, including medical colleges. We contacted a vaccine company and few vials labeled as "For IM/ID use" were sourced from Mumbai (1200 km away from here). We asked the Chief Medical Officer, Shimla district to write a letter to all health facilities around our Hospital to give first aid to animal bite patients and then refer them to DDU Hospital for vaccination. Now we were able to pool the patients and divide a single $1 \mathrm{ml}$ vaccine vial among four patients. After continuous advocacy, our stress that WHO has given its approval for ID use of rabies vaccine and that subsequent approval has been granted by Government of India was enough for doctors to prescribe the vaccine as ID. Last Year, we got ethical approval to inject rabies Immunoglobulins (RIGs) only locally in and around the wound at times of scarcity of RIGs in the market. The subsequent follow up of patients proved life saving in crisis of shortage of RIGs. Due to shortage of RIGs we innovatively vaccinated people bitten by rabid dogs or people who had consumed rabid cow's milk and followed them for outcome, apart from having Rabies Fluorescent Focus Inhibition Test (RFFIT) was done for few of the patiens for verification of protective titers. We innovated a technique of extraction of last drop of vaccine from the vial and also saved a drop of RIGs being used for test dose before giving RIGs to the patients. Results: The first low cost anti-rabies clinic was started on August 2, 2008 after long advocacy sessions with the authorities and the doctors. Since then, we have done many innovations based on local requirements and patients' feedback and accessibility to treatment. We have given pre and post-exposure prophylaxis to more than 12,000 animal bite victims over more than five years period in this single clinic, saving lives as well as money without any failure even in difficult rabid animal bite cases. Our innovation helped us save the vaccine and immunoglobulins till the last drop. Conclusions: Innovative ways by health providers backed by extensive literature review and scientific evidence can help patients get low cost health deliverables that increase their compliance as medicines/vaccines become affordable to them. Third world countries need to innovate their own ways to solve their problems of scanty resources and find innovative solutions to conquer them, rather than looking elsewhere for solutions.

\section{Keywords}

Intradermal Rabies Vaccination, Local RIGs Infiltration, Local Innovations, Rabid Animals, RIGs Scarcity, One Health, Zoonosis

\section{Introduction}

Rabies is a dreaded disease and an estimated 55,000 people die of rabies every year and out of that 31,000 deaths are reported from the Asian continent. About 20,000 deaths are reported from India, i.e. one death every half an hour. Animal bite incidence rate is 17.4 million bites every year. Annual medicinal (vaccines + other drugs) cost for animal bite treatment is $\$ 4$ Million (2003) [1]. Himachal Pradesh is in the North, bordering China, and is predominantly rural and hilly and villages are near forests, where wild reservoirs of rabies exist. Since health facilities are not accessible easily, we need to innovate on existing schedules of rabies vaccination, keeping in view the compliance of the patients and their affordability to give them the best possible option of treat- 
ment. In the year 2006 and 2007, we at DDU Hospital Shimla experienced a severe shortage of rabies vaccine and patients were running from pillar to post to fetch rabies vaccine. At the same time, we learnt that some of the patients died because either they were not able to purchase the vaccine mainly because of the high costs of vaccine, \$35, or they ignored the animal bites and did not seek the Post Exposure Prophylaxis (PEP). Since last year, we have been experiencing non-availability of rabies immunoglobulins (RIGs) in the market and had to innovate to save the lives of the patients.

\section{Methods}

During shortage of rabies vaccine in 2008, we contemplated to start a low cost intra-dermal clinic so as to make vaccine affordable as intramuscular (IM) vaccination cost five times more than ID vaccination. But there were three main hurdles. One hurdle was the non-availability of rabies vaccine vials having written on them "For IM/ID use" and another hurdle was few patients attending the DDU Hospital's anti rabies clinic, sometimes only one or two per day, which was insufficient to open a vaccine vial and distribute among them. The vaccine producing companies were asked to make us available the cell culture rabies vaccine vials labeled as "For IM/ID Use" but none were ready to provide for fear of losses. The third problem being faced was reluctance of the hospital doctors to prescribe ID vaccine as this was not the practice at higher teaching institutions, including medical colleges.

We contacted a vaccine company and few vials labeled as "IM/ID" use were sourced from Mumbai (1200 km away from here). We asked the Chief Medical Officer, Shimla District to write a letter to all health facilities around DDU Hospital to give first aid to animal bite patients and then refer them to DDU Hospital for vaccination. Now we were able to pool the patients and divide a single $1 \mathrm{ml}$ vaccine vial among four patients through pooling strategy [2]. After continuous advocacy, our stress that WHO has given its approval for ID use of rabies vaccine and since subsequent approval has been granted by Government of India was enough for doctors to prescribe it as ID. We innovated a technique of extraction of last drop of vaccine from the vial and also saved a drop of RIGs used for test dose before use.

Last Year, we got ethical approval to inject RIGs only locally in and around the wound at times of scarcity of RIGs in the market and subsequent follow up of patients proved life saving in crisis of shortage of RIGs. Due to shortage of RIGs, we innovatively vaccinated people bitten by rabid dogs or people, who had consumed cow's milk and followed them for outcome, apart from having RFFIT done for few of them for verification of protective titers.

The first low cost anti-rabies clinic started on August 2, 2008 after long advocacy sessions with the authorities and the doctors. This centre was formally launched as State Intra-dermal Anti-rabies Clinic and Research Centre (ARC \& RC) by Mission Director National Rural Health Mission on September 28, 2010. Since then, we have done many innovations based on local requirements and patients' feedback. We have given pre and post exposure prophylaxis to more than 12, 000 animal bite victims over more than five years period, saving lives as well as money of the poor patients and the government.

Some of the innovations that were conceived over the years especially in the light of scarcity of RIGs and keeping in view the compliance of the patient to prophylaxis are detailed as follows:

1) Pooling Technique of Himachal for IDRV to save money and lives by making rabies vaccine affordable.

2) Local RIGs infiltration of wound/s without systemic IM Use for those who cannot afford full dose of RIGs as per body weight or in case of RIGs scarcity.

3) 8 site ID for referred patients who could not get RIGs immediately and for those who need referral to higher institutions for local RIGs using 8-2-2-0-2 schedule instead of 2-2-2-0-2 schedule.

4) 4 site one time boosters for previously vaccinated patients, who do not require RIGs.

5) 4 site pre-vaccination to those who are likely to be unavailable for next dose, like rag pickers, who may need 4 site one time $0.1 \mathrm{ml}$ IDRV boosters without RIGs in case of re-exposure within 1 - 3 years.

6) Pup vaccination before 3 months of age after pup bite/scratch was found to have caused death of four patients in Himachal due to rabies.

7) One week 4-4-4 site PEP for those having consumed rabid cow's raw milk/butter milk in situations of non-availability of RIGs in remote rural areas due to their high dropout for last dose on Day 28.

8) Saving a drop of vaccine and immunoglobulins: we could extract the last drop of vaccine ( $0.2 \mathrm{ml}$ or less) and use it in the next vial. We also used smaller quantities of RIGs than advised for the test dose, while saving 
the RIGs till its last drop and using this drop for single puncture wound/s caused by nail/s of monkeys by shifting the drop to next RIGs vial for use.

9) Testing brain samples of stray animals reported dead to Shimla Municipality for evidence of rabies.

10) Extensive use of WhatsApp to transfer wound images from distant remote places and hold real-time consultation with many stakeholders simultaneously.

11) Development of Information, Education and Communication (IEC) Material in local languages (Poster) to educate public and Development of training manual for Doctors and paramedics.

12) Coordination with forest and animal husbandry departments to map the foci of rabies in affected villages near the forests to plan for oral rabies vaccination baits for wild animals in these forests.

\section{Results}

The animal bite patient data since start of this low cost intra-dermal anti-rabies clinic and research centre (ARC \& RC) is described in the following Table 1.

Since the compliance rate increased to 2.8 times as seen in the initial months of start of the low cost anti-rabies clinic [2], on the whole, we can assume that more than 4302 patients over five years, who could not have complied with the instructions of the doctor to get themselves vaccinated, got the PEP as it became affordable. The affordability of the vaccine helped government take a decision to administer the vaccine free to all in many clinics of the state. As per WHO estimates, every year, more than 15 million people receive post-exposure prophylaxis, mostly in China and India. Post-exposure prophylaxis is thought to prevent more than 270,000 deaths in Asia and Africa [3]. We therefore assume to have saved the life of more than 200 patients and $\$ 281,073$ of money of the patients and the government over the last five years period through this single low cost IDRV clinic. The sudden surge of cases in 2014-15 is due to the fact that RIGs are not available anywhere and even IG Medical College is referring cases to our clinic run in a small Zonal level hospital.

\section{The Innovations Conceived at ARC \& RC DDU Hospital Shimla Himachal Pradesh, India}

\section{1) Pooling technique of Himachal for IDRV}

As described above we pool the vaccine and the patients making vaccine affordable to them [2] and save lives. We ask every patient to purchase a vial of vaccine and divide the same among four of them and keep the remaining three vials to be used on all four patients on subsequent three visits for ID vaccination. Popular saying is that you bring one vaccine vial (\$5) rest we would give "Free" and is very catchy for the patients. More than 12,000 animal bite victims have benefited from this unique pooling methodology in our centre and thousands have benefited all over the state and neighbouring states over the five years period as the technique has became a routine practice all over the state of Himachal. We have spread the technique through trainings to the doctors in neighbouring states as well. We not only divide the $1 \mathrm{ml}$ vaccine vial among four patients but have learnt the method to extract remaining $0.2 \mathrm{ml}$ drop of the vaccine from the vaccine vial through vacuum pressure inside by pushing air into vaccine vial and shifting this vaccine drop to the next vaccine vial to be reconstituted [2]. However at the end

Table 1. Year wise details of the animal bite patients attending ARC \& RC at DDU Hospital Shimla, HP, India.

\begin{tabular}{|c|c|c|c|}
\hline Year & No Of patients & Cost saving & Lives Saved \\
\hline From $2^{\text {nd }}$ August 2008 & 1586 & Rs. 2220400/- [\$37006] & \\
\hline 2009 & 1981 & Rs. 2773400/- [\$46223] & \\
\hline 2010 & 1352 & Rs. 1892800/- [\$31546] & \\
\hline 2011 & 1377 & Rs, 1927800/- [\$32130] & \\
\hline 2012 & 1804 & Rs. 2525600/- [\$42093] & 216 \\
\hline 2013 & 1168 & Rs. 1635200/- [\$27253] & \\
\hline 2014 & 2042 & Rs. 2858800/- [\$47646] & \\
\hline Till April 30 ${ }^{\text {th }}, 2015$ & 736 & Rs. $1030400 /-$ [\$17173] & \\
\hline Total & 12046 & Rs. $16864400 /-[\$ 281073]$ & \\
\hline
\end{tabular}


of the day we discard all remaining vaccine in the reconstituted vial as per the national guidelines issued. This saving of small drops resulted in saving of thousands of rupees and making vaccine available free to those who cannot purchase even a single vial of vaccine like rag pickers. However discarding left out vaccine is huge wastage of resources and we must take forward the efforts and evidence generated by Khawplod P, Wilde H. et. al. [4] [5] to store reconstituted vaccine vials at 2-8 degree centigrade for a week and reuse in resource poor settings like ours which has proved to be economical, immunogenic and is helpful in saving lives in remote inaccessible areas of developing countries like ours.

2) Local RIGs infiltration of wound/s without systemic IM Use for those who cannot afford full dose of RIGs as calculated based on body weight or in case of RIGs scarcity

We are experiencing a severe shortage of rabies immunglobulins (RIGs) all over India for the last one year. There are experimental studies that prove that immunoglobulin must be injected into and around the wounds, whatever goes into muscle or fat elsewhere will not produce a significant circulating level to neutralize the virus at the bite sites [6]-[8]. Rather full systemic infiltration of RIGs have resulted in failures as well [9]. Therefore in situations of non availability of RIGs in the market only local infiltration into and around the wound/s is done without systemic IM use. It has been found to be effective even in suspected rabid dogbite [10] patients as our follow-up of patients for more than nine month period has shown [11]. Also local RIGs are helpful for those, who cannot afford to have, full dose of RIGs as calculated based on body weight, due to its high cost (\$28 eRIGs) and such patients can be given only local infiltration in the wound without systemic IM use after recording their consent. Till April 2015, we have given local infiltration to more than 2178 patients without failure. Some of the patients have been bitten inside mouth or lips or on the face by suspected rabid dogs. We kept unutilized Anti Rabies Serum (ARS) procured from Central Research Institute (CRI) Kasauli for use next day at +2 to +8 degree centigrade in aseptic conditions inside the fridge. As enquired from CRI, the serum is enzyme refined equine rabies immunoglobulins containing preservative Phenol not more than $0.25 \% \mathrm{v} / \mathrm{v}$ and is not to be frozen.

A very interesting case that came to our clinic was 8 years old girl whose 11 yrs old brother was declared dead due to rabies in Medical College (IGMC), Shimla. She came after both of them were bitten by a two and a half month old pup born to their pet bitch. Both bitch and pup died sometime ago and her brother died 2 months after he was bitten on hands. The girl was also bitten on right hand by the same rabid pup and was brought to IGMC Shimla. After two ID shots she was referred to our Anti Rabies Clinic and Research Centre (ARC \& RC).We injected the $8 \mathrm{~cm}$ scar on the Rt. hand with RIGs allover and gave her 8 site IDRV for early immunity and took blood sample for RFFIT (D 8 titers $12.5 \mathrm{IU} / \mathrm{ml}$ ). The girl is still surviving after one year of local RIGs as she had 35 - 57 percent chances of death due to rabies [12]-[15]. Pups can be rabid and infect people further strengthens our resolve to vaccinate pups before bringing them home even if they are less than three months of age in rabies endemic countries in Asia \& Africa to avoid rabies [16]. Also a recent finding further confirms this aspect of pup being rabid at young age of 9 weeks [17].

\section{Further Scaling Up:}

We intend to continue purchase few available eRIG vials from CRI Kasauli and give eRIGs to patients locally in and around the wound free of cost till eRIGs are not available in the market. Thereafter we would pool the poor patients and the RIGs vials purchased by them like we do for rabies vaccine and share the RIGs vials amongst them like pooling technique of Himachal for IDRV [2]. Those who would like to purchase the full calculated dose as per WHO protocol shall be given RIGs accordingly but those who express their inability to do so would be given RIGs only locally after getting explicit consent from them, till WHO guidelines are amended.

\section{3) 8 site ID for referred patients who could not get RIGs immediately}

When patients are referred from for away places to our clinic for RIGs with one ID (2 site) or IM dose already given and when RIGs are not immediately available at our clinic as well, we give 8 site ID vaccination to boost the immune response [18]. As described above, in another case we took the serum sample for testing protective titers on day 6 that had antibodies titers $3.5 \mathrm{IU} / \mathrm{ml}$ (RFFIT) after first IM dose and 8 site ID dose at day 3. Mostly such cases that are referred to our clinic after one IM or ID dose in the field are given 8-2-2-0-2 at our clinic along with only local RIGs which is sufficiently protective as shown by the subsequent follow-up of such patients and protective antibody titers are achieved early on days 6 - 8. We have instructed our field officers to give 8 site IDRV consuming full $1 \mathrm{ml}$ vaccine vial and then refer the patient to higher institution for RIGs as RIGs are not available in the field. 8 site IDRV is the best a field staff can do in remote rural areas amidst RIGs scarcity in the field activating all major lymph node sites for faster immune response and consuming the entire 1 
$\mathrm{ml}$ vaccine vial, without vaccine wastage.

4) $\mathbf{4}$ site boosters for previously vaccinated patients without RIGs

Since we are facing an acute shortage of RIGs for the last one year, we give only 4 site $0.1 \mathrm{ml}$ Cell Culture Rabies Vaccine (CCRV) in one setting for previously vaccinated persons currently having type III wound/s without any RIGs and that has proved to be efficacious in many studies [19]. There is strong evidence that patients previously vaccinated with CCRV need only 4 shots of $0.1 \mathrm{ml}$ IDRV in one sitting even if they are exposed to suspected rabid animals and WHO recommends such a practice [20]. We recorded 59 such patients who had CCRV previously and were given one time 4 site $0.1 \mathrm{ml} \mathrm{CCRV} \mathrm{without} \mathrm{RIGs.} \mathrm{Blood} \mathrm{samples} \mathrm{were}$ drawn from two such patients that showed (RFFIT) titers of $8.9 \mathrm{IU} / \mathrm{ml}$ and $12.6 \mathrm{IU} / \mathrm{ml}$ at day 14 which are sufficiently protective. The four sites selected were two deltoid and two suprascapular skin that were found to be acceptable to Indian populations.

5) 4 site Pre Exposure Prophylaxis (PrEP) to those who are unlikely to be available for next dose like rag pickers

We tried to give pre exposure vaccination to rag pickers to protect them from the threat of rabies as per WHO protocol on days $0,7,28$; but many of them were not available for vaccination after first or second dose. We then followed a method of one time 4 site $0.1 \mathrm{ml}$ vaccination on both deltoids and both suprascapular skin which proved acceptable and immunogenic on future boosters [21]. Though more studies are required to establish this schedule but still something is better than nothing for such vulnerable populations that are careless and are not aware of consequences of animal bites. Potent vaccines need to be used in such precarious situations where only one time prophylaxis is to be given (vaccine we used had titers $7.18 \mathrm{IU} / \mathrm{vial}$ ). More than $700 \mathrm{rag}$ pickers have been given such 4 site onetime pre exposure prophylaxis till date. Such people would require only one time 4 site $0.1 \mathrm{ml}$ IDRV boosters without RIGs in case of re-exposure within 1 - 3 years.

6) Pup vaccination before 3 months of age after pup bite/scratch were found to have caused four deaths in Himachal due to rabies among patients

The pups in India are not vaccinated before three months for fear of maternal antibodies interfering with the active immunity leading to insufficient immune responses. The Indian veterinarians claim to follow Centre for Disease Control and Prevention (CDC) guidelines on pup vaccination [22]. Though India, unlike USA, is rabies endemic country and we need to keep in mind the deaths due to rabies caused by pup bites, while following CDC guidelines. Many studies indicate that puppies of vaccinated bitches have no immunity conferred by their mothers [23]. Rather healthy looking dogs are positive for rabies antigen and can be dangerous [24]. The author while investigating four deaths due to rabies found that deaths due to pup bite or scratch are common [16] rather a two and a half month old pup that was found dead in Shimla city in 2014 was sent for brain examination to Central Research Lab (CRI) Kasauli in Himachal Pradesh, India and was found to be positive for rabies virus through Florescent Antibody Test (FAT). Thereafter a recommendation was made to the Government of Himachal through Pr. Chief Secretary (Animal Husbandry) to examine the matter and give directions to start vaccination of pups against rabies at very first contact with humans even if they are below three months of age and then give them booster at three months and yearly thereafter.

For stray pups the newly developed oral dog vaccines [25]-[27] have shown promising results and even puppies of age 7 - 10 week old have been successfully vaccinated with the oral vaccine [28] and even with injectable rabies vaccines [29].

7) One week 4-4-4 site PEP for those having consumed rabid cow's raw milk/butter milk in situations of non availability of RIGs

Since start of ID clinic in 2008, there are many incidents when we have to give PEP to children in schools for exposure to raw milk/butter milk of rabid cow or a mid day meal cook in school having died of rabies after rabid dog bite etc. Some of the schools were in remote areas about $100 \mathrm{KM}$ away from here. We gave 2 site modified Thai schedule IDRV to school children (Figure 1) but many of the children dropped out for the last dose on Day 28 due to mild side effects of itching, redness etc and due to fear of needle. No RIGs were available. The children were followed and no death was reported even after more than 5 years of follow up.

It has been observed in the last over five years, that dropout for vaccination, especially for Day 28, is more than $90 \%$ in such cases of raw milk consumption from a rabid cow and is consistent with many studies done under rural settings in India [30]. The dropout is mostly due to the fact that such patients usually come from distant remote areas and there is no visible wound that could make them feel the need for repeat vaccination for long time apart.

We come across 40 - 50 cases of raw milk/butter milk consumption of rabid cows/buffalos every year as 


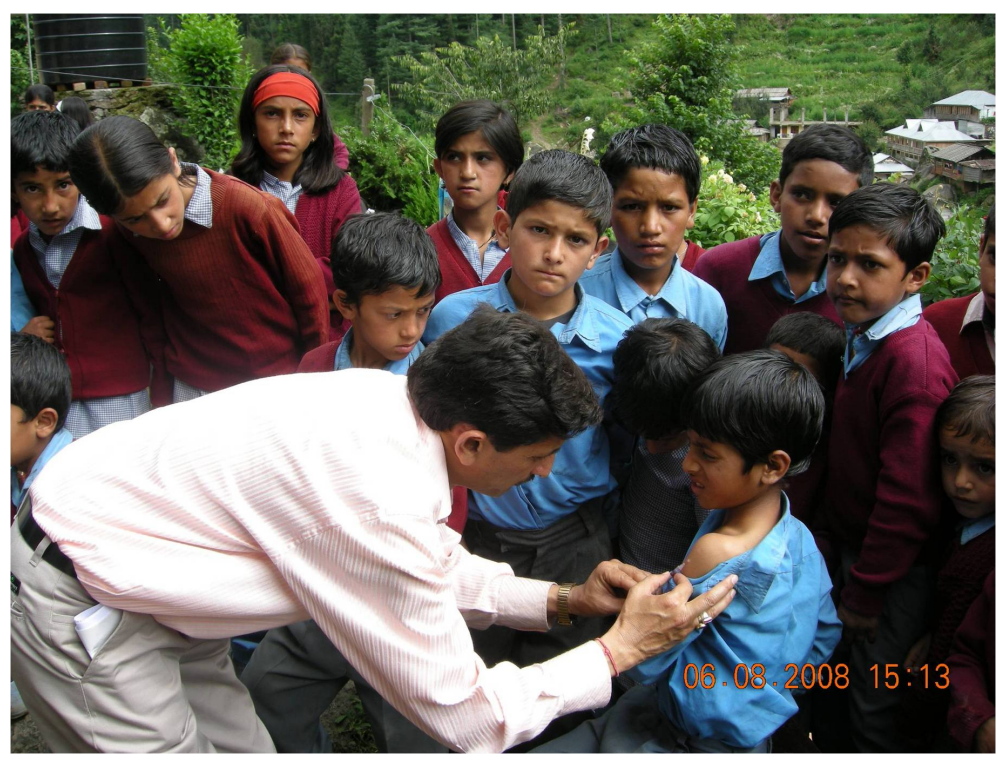

Figure 1. School Children in remote village being given PEP in the year 2008.

livestock is the main livelihood in rural areas specially those nearer to forests. Guidelines [31] suggest that such people need systemic IM RIGs but RIGs are either not available in remote rural areas or not affordable as usually many members of the same family (5 - 6) are affected and each family has to pay more than Rs. 5000 (\$100) for eRIGs only apart from paying for rabies vaccine.

Although scientific literature is scant (regarding transmission of rabies through milk) and in the few studies cited, mammary tissue from rabid animals tested negative [32] but still treatment is suggested regardless of test results. Over the past 15 years, CDC has received mammary tissue and unpasteurized milk from approximately one rabid cow per year. To date, no rabies virus antigen or nucleic acids have been detected [33].

This year, since January till mid May 2015, we have got 35 such patients who had consumed raw milk of cows that died due to rabies. In two separate incidents in June month, we got 112 and 62 patients who had raw milk ingested. In village Sainj (Rural Shimla) 112 people were given 4 site PEP on days 0, 3, 7 as the two rabid dogs had bitten a cow that died of rabies as confirmed by the veterinarian. One dog was killed and the other could not be traced. In second incident in nearby village Punder 62 people who had consumed raw buttermilk were given 4 site one week PEP schedule. The dog was killed and the bitten cow died of clinical rabies. All patients are from remote villages near forests. In times of non availability of RIGs and huge drop out of the patients from remote areas on day 28 we thought of new initiative. Without administration of RIGs, we gave new one week 4-4-4 site prophylaxis that has shown promising results in clinical trials [30] in India. Subsequent follow up has shown that no one had died and 4 site IDRV is well accepted.

8) Saving a drop of vaccine and immunoglobulins (RIGs)

As described in the pooling technique of Himachal for IDRV above, we extract the last drop of leftover vaccine and use it after transferring this drop to the next vaccine vial. We learnt this during shortage of vaccine in the market. Nowadays when RIGs are in shortage we thought of saving even a single drop of RIGs by changing the way we dilute RIGs for test dose before use. Test dose before RIG administration has been made mandatory by the companies in India. Contrary to WHO guidelines and National Guidelines on rabies prophylaxis [34], all manufacturers of RIGs, including, government run CRI Kasauli from where we are sourcing RIGs, stress for giving a test dose of eRIGs before use. The recommendation is to dilute $0.1 \mathrm{ml}$ of RIGs with $0.9 \mathrm{ml}$ of Normal Saline (NS) and give $0.1 \mathrm{ml}$ of this dilution as test dose to ascertain sensitivity. This used to result in wastage of a drop of RIGs, which cannot be tolerated in a situation of non availability of RIGs in the market. To comply with the guidelines of the manufacturers, we thought of taking 1 unit of RIGs in 40 unit insulin syringe and dilute it with 9 units of NS to achieve the same concentration of RIGs as advised by the manufacturer for the test dose. This resulted in saving of $0.075 \mathrm{ml}$ of RIGs that can be used on three patients having single puncture wound mostly caused by the nail of the monkeys. We have seen that such patients usually require only $0.025 \mathrm{ml}$ (1 unit) of RIGs to infiltrate the single puncture wound. 


\section{9) Testing brain samples of stray animals reported dead to Shimla Municipality for evidence of rabies}

Every day we get 8 - 10 patients bitten by stray animals in Shimla Municipality area. In view of the animal right activists, it is difficult to kill a suspected rabid dog/animal for getting its brain sample tested for evidence of rabies. We thought of a plan to extract the brain of animals being reported dead to Municipality while disposing their dead bodies. Animals reported as recently dead, were selected and sent for evidence of rabies to CRI Kasauli. Brain samples of six such animals were sent last year and one dog this year in 2015. The animals tested last year were a Pup, a Monkey, a Langoor, a Himalayan Palm Civet and two dogs (Table 2). All animals tested positive for rabies by Fluorescent Antibody Test (FAT) and/or Biological test (BT) by the CRI Kasauli Lab. This year also (2015), a suspected rabid dog, on biting spree, turned out to be positive for FAT. This is for the first time we were able to prove that biting animals in Shimla Municipality including Monkeys are not free from rabies virus. And patients bitten by stray or wild animals in the municipality need to be given PEP with utmost care since most of the bites are by stray dogs and wild monkeys.

10) Extensive use of WhatsApp to transfer wound images from distant remote places and hold realtime consultation with many stakeholders simultaneously

Extensive use of WhatsApp is being done to transmit the photographs/images of the wounds for consultations on local RIGs. Also real-time consultations are being done to discuss the matter among many stakeholders over WhatsApp. In one such incident the image was sent of a wound caused by monkey bite on the forearm of a patient, tendon was visible inside on the floor of the gaping wound and need not to be punctured to avoid paresis. Thereafter it was advised that RIGs may be sprayed over the visible surface of the tendon and RIGs were infiltrated all over the edges of the wound keeping the bevel of the needle towards the wound.

\section{1) Development of IEC Material}

A poster for public and a manual for Doctors and paramedics were developed: it was felt that minimum basic first aid is neglected by the majority of patients due to ignorance of its life saving value. Therefore a poster [35] in English and local language Hindi was developed to make people aware of the management of animal bite wound at their level as first aid. Now the poster is being translated toother Indian languages as well. We have also developed a new one page Matrix as ready reference to understand various innovations and different schedules of vaccination in different situations, especially that caused by the unavailability of RIGs. The new Matrix was extensively discussed in a recent workshop of District level master trainers held on May 27 - 28, 2015. This became urgent in the wake of ongoing scarcity of RIGs in the state. A training manual was developed to train the doctors and paramedics in 2010 and a new manual is under preparation keeping in view the local conditions and innovations that emerged especially in a situation of unavailability of RIGs.

12) Coordination with forest and animal husbandry departments to map the foci of rabies in affected villages near the forests to plan for oral rabies vaccination baits for wild animals in these forests

We are in the process of identifying wild rabies foci in the forests in Himachal Pradesh so that a joint campaign can be launched along with the forest and animal husbandry departments to drop rabies vaccine baits for wild animals to control rabies spillover to nearby villages as a ONE Health initiative. To achieve this objective of ONE Health we are mapping the inflow of rabid dog bite patients all over the state and analyzing reports from animal husbandry department on deaths of livestock due to rabies. Animal Birth Control (ABC) programme need to be further strengthened in Municipalities of the state. Some of the foci identified are forests near Theog,

Table 2. Analysis of brain samples proves that biting animals in Shimla Municipality including Monkeys were not free of rabies virus despite a long history of Animal Birth Control (ABC) programme as the result detailed below reveal:

\begin{tabular}{|c|c|c|c|c|c|}
\hline Sr. No. & Animal & Age & $\mathbf{F A T}+/-$ & $\mathbf{B T}+/-$ & Remarks \\
\hline 1 & Pup & 2.5 months & + ve & Not Done & The pup was found dead near Raj Bhawan Forest MC Shimla \\
\hline 2 & Dog & Adult & $+\mathbf{v e}$ & + ve & The dog was found dead at Sanjauli MC Shimla \\
\hline 3 & Dog & Adult & + ve & + ve & The dog was found dead at Chaura Maidan Shimla \\
\hline 4 & Monkey & Adult & -ve & + ve & The monkey was found dead at the Mall Road MC Shimla \\
\hline 5 & Langoor & Adult & $+\mathbf{v e}$ & $+\mathbf{v e}$ & The Langoor was found dead at Middle Bazaar MC Shimla \\
\hline 6 & Himalyan Palm Civet & Adult & $+\mathbf{v e}$ & $+\mathbf{v e}$ & The Civet was found dead at the Mall Road MC Shimla \\
\hline
\end{tabular}


Manali, Nadaun, Shimla Municipality, Rampur, Chopal, Sangrah, Chamba, Arki, Panjgain and Dharamsala.

\section{Conclusion}

Innovative initiatives by health providers backed by extensive literature review and scientific database can help patients get low cost health deliverables that increase their compliance as medicines/vaccines become affordable to them. Third world countries need to innovate their own ways to solve their problems of scanty resources and find innovative solutions to conquer them rather than looking elsewhere for solutions.

\section{Recommendations}

We have learnt from our experience after more than 90,000 vaccine inoculations and more than 2000 local RIGs injections that both the vaccine and the rabies equine immunoglobulins/serum are nowadays very safe, refined, potent and effective and medical fraternity need not fear any adverse events. Since last year only two mild adverse events were recorded with both the vaccine and the RIGs and were managed well with inj. Avil and Tab cetrizine. Also the rabies prophylaxis in scant resources is the problem of Asia and Africa that can be resolved with the help of experimental studies conducted by our fellow researchers abroad and in India. We need to choose a methodology, best suited to our circumstances to help benefit the poor, the most, keeping compliance issues in mind. Integrated ONE Health approach is must if we want to make Himachal Pradesh first Rabies Free State of India by 2020. A Zoonotic division needs to be created independently in developing countries to control Zoonosis effectively including rabies.

\section{Acknowledgements}

Our sincere thanks to Dr. S. N. Madhusudana, Professor of Neurovirologyand Head WHO Collaborating Centre for Reference and Research on Rabies, Department of Neurovirology, NIMHANS, Bangalore, India for his technical approval of the local rabies imunoglobulins study and testing of serum samples for RFFIT free of cost. Our thanks to Dr. David .C. Anderson for his thought provoking article titled WHO guidelines dealing with immunoglobulin use impede rabies prevention, Asian Biomedicine Vol. 1 No. 1 June 2007. Our Thanks to the Retired Senior Medical Superintendents of Deen Dayal Upadhyaye Hospital (DDU), Dr. P.L. Gaunta who encouraged us for starting local RIGs in our rabies research centre and Dr.Jitender Kumar who helped us start the low cost Intra-dermal Anti Rabies Clinic in initial phases. Thanks to Dr. Ashok Kale for sensitizing political leadership of the state convincing them of usefulness of Intra-dermal method of rabies vaccination.

Ethical clearance for local RIGs use was taken from the Institutional Ethics Committee of Jaypee University of Information Technology in a meeting held on $23^{\text {rd }}$ May, 2014 and was assigned the code as IEC/Project no. 11-2014. Our thanks to the Ethical Committee members of J.P. University, who realized the urgency of such a study in circumstances of non-availability of RIGs in the market and granted approval. Our sincere thanks to all ARC\&RC staff that helped us do the study especially our thanks to staff nurse posted in the clinic Mrs. Nirmal Gupta for all her efforts and innovations in giving RIGs locally and to Female Health Worker Mrs. Dropda Shyam for her contributions to develop pooling strategy of intra-dermal anti-rabies vaccination. Thanks to animal husbandry department of Himachal Pradesh for extracting animal brains and Forest department for agreeing to map rabies foci in the state.

Many thanks to Dr. Henry Wilde, Dr. Mary J. Warrell, Dr. M. K. Sudershan, Dr. Ashwath Narayana and Dr. B.J. Mahendra for their trainings, moral support and guidance. Our thanks to all authors whose references we have quoted in our studies referred here; in fact every one of them has contributed to the noble cause.

Thanks to Cadila Healthcare Ltd. (Zydus Fortiza) for sponsoring same Batch (CM 132) of 480 vaccine vials for the local infiltration of RIGs study.

And lastly our thanks to Central Research Institute, CRI Kasauli for making few vials of ARS/RIGs available in crisis of RIGs shortage all over and for testing animal brains for FAT/BT free of cost. Our thanks to the Government of Himachal Pradesh and National Health Mission for giving all administrative support and logistics especially funds for training medical manpower and for printing manual and matrix for wider dissemination.

\section{Conflict of Interest}

None declared. 


\section{References}

[1] Sudershan, M.K. (2004) Assessing Burden of Rabies in India WHO Sponsored National Multi-Centric Survey 2003. Association for Prevention and Control of Rabies in India, AMS Code 1079420.

[2] Bharti, O., Damme, W., Decoster, K., Isaakidis, P., Appelmans, A., Ramachandran, V. and Phull, A. (2012) Breaking the Barriers to Access a Low Cost Intra-Dermal Rabies Vaccine Through Innovative "Pooling Strategy”. World Journal of Vaccines, 2, 121-124. http://dx.doi.org/10.4236/wjv.2012.23016

[3] WHO Report (2015) More than 15 Million Post-Exposure Prophylaxis Every Year. http://www.who.int/rabies/human/situation/en/

[4] Kamoltham, T., Khawplod, P., Wilde, H., et al. (2002) Rabies Intradermal Post-Exposure Vaccination of Humans Using Reconstituted and Stored Vaccine. Vaccine, 20, 3272-3276. http://www.ncbi.nlm.nih.gov/pubmed/12213396 http://dx.doi.org/10.1016/S0264-410X(02)00299-2

[5] Khawplod, P., Tantawichien, T., Wilde, H., et al. (2002) Use of Rabies Vaccines after Reconstitution and Storage. Clinical Infectious Diseases, 34, 404-406. http://www.ncbi.nlm.nih.gov/pubmed/11774089 http://dx.doi.org/10.1086/324507

[6] Wilde, H., Shantavasinkul, P., Hemachudha, T., et al. (2009) New Knowledge and New Controversies in Rabies. Journal of Infectious Diseases and Antimicrobial Agents, 26, 63-74. http://www.idthai.org/Publication/pdf/Vol26-2/p.63-74.pdf

[7] Chomchay, P., Khawplod, P. and Wilde, H. (2000) Neutralizing Antibodies to Rabies Following Injection of Rabies Immune Globulin into Gluteal Fat or Deltoid Muscle. Journal of Travel Medicine, 7, 187-188. http://www.ncbi.nlm.nih.gov/pubmed/11003730 http://dx.doi.org/10.2310/7060.2000.00057

[8] Anderson, D.C. (2007) WHO Guidelines Dealing with Immunoglobulin Use Impede Rabies Prevention. Asian Biomedicine, 1. http://abm.digitaljournals.org/index.php/abm/issue/view/18/showToc

[9] Wilde, H., Sirikawin, S., Sabcharoen, A., et al. (1996) Failure of Postexposure Treatment of Rabies in Children. Clinical Infectious Diseases, 22, 228-232. http://cid.oxfordjournals.org/content/22/2/228.full.pdf

[10] Agarwal, A.K., Mahore, R.N., et al. (2014) Why a Dog Needs to Be Observed for 10 Days after Biting a Human Being? APCRI Journal, XV, 6. http://apcrijournal.org/JournalPDF/January2014.pdf

[11] Omesh Bharti, K., Madhusudana, S.N., et al. (Under Review) Local Infiltration of Rabies Immunoglobulins without Systemic Administration: An Alternative cost Effective Approach for Passive Immunization against Rabies.

[12] Veeraraghavan, N. (1971) Annual Report of the Director 1969 and Scientific Report 1970. Coonoor: Pasteur Institute of Southern India.

[13] Fearon, W.R. (1934) An Introduction To Biochemistry. William Heinemann (Medical Books) Ltd., London, 109. http://ir.nmu.org.ua/bitstream/handle/123456789/17174/2872daf9545154aa97ec8aff71fc907c.pdf?sequence=1\&isAllo wed $=\mathrm{y}$

[14] Topley, W.W.C. and Wilson, G.S. (1936) The Principals of Bacteriology and Immunity. 2nd Edition, Edward ARNOLD \& Co., London, 1510.

[15] Cornwall, J.W. (1923) Statistics of Antirabic Inoculations in India. BMJ, 2, 298. http://dx.doi.org/10.1136/bmj.2.3268.298

[16] Bharti, O., Ramachandran, V., Kumar, S. and Phull, A. (2014) Pup Vaccination Practices in India Leave People to the Risk of Rabies_Lessons from Investigation of Rabies Deaths Due to Scratch/Bite by Pups in Remote Hilly Villages of Himachal Pradesh, India. World Journal of Vaccines, 4, 7-10. http://dx.doi.org/10.4236/wjv.2014.41002

[17] 15 Treated after Vt. Puppy Tests Positive for Rabies. http://www.wcax.com/story/23772297/15-treated-after-vt-puppy-tests-positive-for-rabies

[18] (2000) Intradermal Application of Rabies Vaccines, Report of a WHO Consultation, Bangkok, 4. http://www.who.int/rabies/en/Intradermal_application_of_rabies_vaccines.pdf

[19] Shantavasinkul, P., Tantawichien, T., Jaijaroensup, W., et al. (2010) A 4-Site, Single-Visit Intradermal Postexposure Prophylaxis Regimen for Previously Vaccinated Patients: Experiences with $>5000$ Patients. Clinical Infectious Diseases, 51, 1070-1072. http://cid.oxfordjournals.org/content/51/9/1070.full.pdf+html

[20] Rabies Pre and Post Rabies Pre and Post-Exposure Prophylaxis in Humans exposure Prophylaxis in Humans. http://www.who.int/rabies/PEP_prophylaxis_guidelines_June10.pdf?ua=1

[21] Bharti, O. (2015) Immunizing Vulnerable Populations Like Rag Pickers, Garbage Collectors, Municipality Workers and Newspaper Hawkers against Rabies in Shimla Municipality, HP, India. World Journal of Vaccines, 5, 19-24. 
http://dx.doi.org/10.4236/wjv.2015.51003

[22] (2013) Rabies Vaccination, Centre for Disease Control and Prevention, CDC 24x7; Saving Lives. Protecting People ${ }^{\mathrm{TM}}$. http://www.cdc.gov/rabies/specific_groups/veterinarians/vaccination.html

[23] Pimburage, R.M.S., Gunatilake, M., et al. (2013) Comparison of Immunogenicity to Anti-Rabies Vaccine in Dogs of Different Age Groups. Proceedings of the 4th Rabies in Asia Conference: RIACON 2013, Bangkok, 11-13 September 2013, 46. http://www.rabiesinasia.org/Scientific\%20programme.pdf

[24] Garba A., Umoh, J.U., et al. (2015) Rabies Virus Neutralizing Antibodies in Unvaccinated Rabies Occupational Risk Groups in Niger State, Nigeria. International Journal of Tropical Disease \& Health, 6, 35-43. http://www.sciencedomain.org/abstract.php?iid=852\&id=19\&aid=7650 http://dx.doi.org/10.9734/ijtdh/2015/14461

[25] Cliquet, F., Gurbuxani, J.P., Pradhan, H.K., Pattnaik, B., Patil, S.S., Regnault, A., Begouen, H., Guiot, A.L., Sood, R., et al. (2007) The Safety and Efficacy of the Oral Rabies Vaccine SAG2 in Indian Stray Dogs. Vaccine, 25, 3409-3418. file:///C:/Users/a/Downloads/Vaccine\%20paper.pdf

[26] Darkaoui, S., Boué, F., Demerson, J.M., et al. (2014) First Trials of Oral Vaccination with Rabies SAG2 Dog Baits in Morocco. Clinical and Experimental Vaccine Research, 3, 220-226. http://www.ncbi.nlm.nih.gov/pmc/articles/PMC4083075/

[27] Oral Immunization of Dogs against Rabies; Report of the 6th WHO Consultation; World Health Organisation. http://www.who.int/rabies/en/Oral_immunization_of_dogs_against_rabies_report_6th_WHO_consultation.pdf

[28] Schumacher, C.L., Hammami, S., Chaparro, F., Bishop, G., von Teichman, B.F., Aubert, M.F.A., Bingham, J., Cliquet, F. and Aubert, A. (1997) Safety of the Oral Rabies Vaccine SAG2 and Efficacy of Its Delivery System DBL2 in Indigenous Dogs. http://searg.info/fichiers/articles/1997163174L.PDF

[29] Morters, M.K., McNabb, S., Horton, D.L., Fooks, A.R., Schoeman, J.P., Whay, H.R., Wood, J.L.N. and Cleaveland, S. (2015) Effective Vaccination against Rabies in Puppiesin Rabies Endemic Regions. Veterinary Record. http://veterinaryrecord.bmj.com/content/early/2015/06/24/vr.102975.full.pdf+html

[30] Sudarshan, M.K., Narayana, D.H.A., Madhusudana, S.N., et al. (2012) Evaluation of a One Week Intradermal Regimen for Rabies Post-Exposure Prophylaxis: Results of a Randomized, Open Label, Active-Controlled Trial in Healthy Adult Volunteers in India. Human Vaccines \& Immunotherapeutics, 8, 1077-1081. http://www.ncbi.nlm.nih.gov/pmc/articles/PMC3551879/ http://dx.doi.org/10.4161/hv.20471

[31] Association for Prevention \& Control of Rabies in India (APCRI) (2009) Manual on Rabies Immunoglobulin (RIG) Administration. 14. http://rabies.org.in/rabies/wp-content/uploads/2009/11/Manual-on-Rabies-Immunoglobulin-Administration.pdf

[32] Carl Armstrong in a Personal Communication on Promed Mail Downloaded from Google. http://www.commed.vcu.edu/IntroPH/Zoonoses/rabiesandcowsmilk01056.htm

[33] The National Association of State Public Health Veterinarians (NASPHV), 2011 Revision of the Compendium of Animal Rabies Prevention and Control, 2. http://www.nasphv.org/Documents/RabiesCompendium.pdf

[34] (2003) National Guidelines on Rabies Prophylaxis. 10. http://www.nicd.nic.in/Rabies guidelines2014.pdf

[35] Poster Developed for IEC.

http://rabiesalliance.org/uploads/media/Resources_Asia/Asia_written_in_English/13._Rabies_English_Simple_1.pdf 